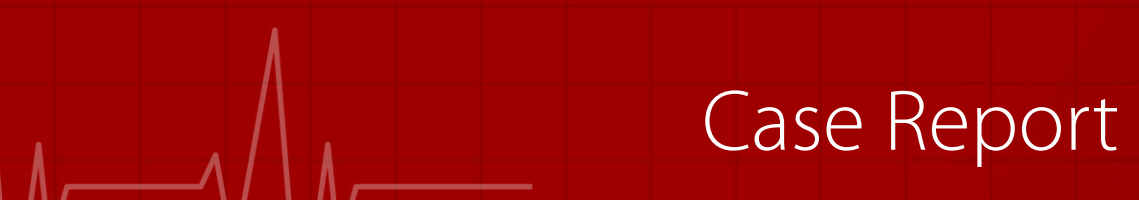

Journal of Emergency Medicine Case Reports

\title{
A rare cause for sciatalgia: Piriformis syndrome
}

Ilker Akbas' ${ }^{1}$, Abdullah Osman Kocak², Alpaslan Unlu ${ }^{3}$, Sinem Doğruyol ${ }^{4}$, Sultan Tuna Akgol Gur ${ }^{2}$ 'Department of Emergency Medicine, Bingöl State Hospital, Bingol, TURKEY

${ }^{2}$ Department of Emergency Medicine, Faculty of Medicine, University of Ataturk, Erzurum, TURKEY

${ }^{3}$ Department of Emergency Medicine, Recep Tayyip Erdogan University, Education and Research Hospital, Rize, TURKEY

${ }^{4}$ Department of Emergency Medicine, Manisa Merkez Efendi State Hospitai, Manisa TURKEY

\begin{abstract}
The clinical symptoms comprising piriformis syndrome emerge as a result of the compression of the sciatic nerve due to anatomical variations or pathological conditions related to the piriformis muscle. The underlying cause in $6 \%$ of cases of lumbar pain, piriformis syndrome might be also defined as entrapment neuropathy of the sciatic nerve. While piriformis syndrome is not rare, it is not well known. As the clinical symptoms may be misleading, it can easily be overlooked, or lead the clinician to an incorrect diagnosis (such as discopathy). In this report, our objective was to present the case of a 18-yearold female patient who presented to the emergency department with sciatalgia and received a diagnosis of piriformis syndrome caused by pyomyositis of the piriformis muscle. The patient arrived at the emergency department reporting severe pain and paresthesia in the right pelvis, thigh, and leg with the inability to walk due to pain. Vital signs were normal except for a high fever. Her Lasègue test was positive, and the patient had severe pain with both internal and external rotation. After radiological examination, the patient was diagnosed with an abscess in the piriformis muscle due to pyomyositis. It was determined that the cause of sciatalgia was piriformis syndrome, with the abscess in the piriformis muscle compressing the sciatic nerve. The abscess was drained after the patient was admitted to the hospital; the patient was then discharged without further complication. The need for high-cost therapeutic methods or even death may result in instances where piriformis syndrome is overlooked by emergency department clinicians, or when it is given the misleading diagnosis of sciatalgia.
\end{abstract}

Keywords: sciatalgia,pyomyositis, piriformis syndrome,piriformis abscess,differential diagnosis

\section{Introduction}

Sciatalgia, a clinical symptom occurring as a result of the sciatic nerve being compressed at any point along its path, is characterized by pain spreading through the lumbar and sacral dermatomes ${ }^{1}$. Piriformis syndrome is seen as a result of the piriformis muscle compressing the sciatic nerve due to anatomic variation or its pathologies. In other words, piriformis syndrome can also be defined as an entrapment neuropathy of the sciatic nerve $e^{2,3}$. Here, we aimed to present a case admitted to the emergency department with sciatalgia for which the diagnosis was piriformis syndrome due to piriformis muscle pyomyositis discovered via clinical examination and radiological imaging.

\section{Case Report}

An 18-year-old woman was admitted to the emergency department reporting severe pain in her right hip and right thigh and leg and inability to walk due to numbness and pain. She stated that the pain started in her hip and spread to her right leg, gradually increasing in intensity through the past couple of days. Three days previously, the patient had been started on an analgesic and myorelaxant oral tablet at another health center, with lumbar discopathy being the general diagnosis as the cause of the pain. There was no history of systemic disease, recent trauma, or intramuscular injection in her medical record. The vital signs of the patient upon admission were normal except for high fever $\left(38.8^{\circ} \mathrm{C}\right)$. In her physical examination, there was minimal tenderness to palpation in the right gluteal region, but no other signs of inflammation. Her Lasègue test was positive (that is, the patient could not raise her leg to a 45-degree angle without pain). While the patient brought her thigh to flexion and extension with ease, she felt severe pain during external rotation and especially internal rotation. Lower extremity peripheral pulses were equally palpable using the hands. The patient had no complaints related to her left gluteal region or left leg. Rectal and other systemic examinations showed no abnormalities. Her laboratory tests were as follows: white blood cell count (WBC): 17,000/ $\mathrm{MM}^{3}$, creatine kinase (CK): $500 \mathrm{U} / \mathrm{L}$. Other parameters were normal.

Tomography was performed in the pelvic area because of the increased sensitivity in the patient's gluteal region; 
heterogeneity consistent with an abscess was present in the piriformis, gluteus maximus and gluteus minimus muscles on the right. Subsequent to tomography, MR imaging was performed to determine the source of abscess formation and to explain the sciatic pain. A lesion that was hypointense and fat-suppressed in the T1-weighted images and showing a significant signal increase in the T2-weighted images was seen inside the right gluteus maximus, minimus and piriformis muscles in the MR. The appearance was interpreted as an abscess inside the piriformis muscle and as sciatic nerve compression not related to the abdomen. The patient was believed to have piriformis syndrome caused by an abscess inside the piriformis muscle. The patient underwent abscess drainage accompanied by tomography and was started on prophylactic antibiotherapy. Staphylococcus aureus grew in the abscess culture that was done. The patient's condition did not regress during follow-up appointments; she underwent surgery and the abscess was cleaned and closed after surgical drainage. Upon resolution of the patient's condition during follow-up appointments, she was discharged with follow-up recommendations after 20 days of hospitalization.

\section{Discussion}

Piriformis syndrome is the underlying cause in about $6 \%$ of those suffering from lower back pain; it is not uncommon, but remains a little-known disease ${ }^{4}$. Piriformis syndrome is seen more commonly in women; the fourth through sixth decades of life constitute the highest risk period for the disease $^{5}$. Piriformis syndrome is easily overlooked due to its clinical symptoms being misleading and directing the physician to incorrect diagnoses (such as discopathy) $)^{5,6}$. Just as piriformis syndrome can occur due to soft tissue inflammation or muscle spasm due to trauma, overuse of the muscle (such as when engaging in exercise involving long walks or running) can also lead to the syndrome ${ }^{5}$. For this reason, piriformis syndrome is often overlooked in athletes and those practicing sports.

After starting inside the pelvis, in the front side of the 2nd to 4th sacral vertebrae, the piriformis muscle goes through the greater ischiatic foramen and ends at the femur trochanter major ${ }^{7}$. In the pelvic region, neurovascular structures pass under or over the piriformis muscle in the foramen ischiadicum majus. The sciatic nerve usually extends underneath the muscle $e^{6,8}$.

Pyomyositis caused by the piriformis muscle leads to irritation and compression in the sciatic nerve; this condition is one of the rare causes of piriformis syndrome 9 . Pyomyositis is a primary pyogenic infection of the skeletal muscle that usually progresses to abscess formation ${ }^{10}$ and is specific only to muscle tissue. It is believed to develop secondary to injuries in the muscle during an asymptomatic and undi- agnosed, transient bacteremia and is not related to bone or soft tissue infections around the muscle ${ }^{8}$. Many patients with pyomyositis are admitted with a clinical picture that changes depending on the location and severity of the infection, but is insidious nonetheless ${ }^{10}$.

The three stages of pyomyositis progress from diffuse inflammation to focal abscess formation and the potential for sepsis ${ }^{10}$. While there is minimal inflammation during the first stage, there is no pus. The second stage is the suppurative stage, which appears 10 to 21 days after symptoms begin. In the last stage, systemic complaints surface along with high fever and septicemia ${ }^{11}$. Delays in the diagnosis of the disease lead to extended hospital stays and a mortality rate around $10 \%{ }^{12}$. In our case, sciatic pain was accompanied by high fever and leukocytosis, and thus we evaluated it as stage third.

Despite the pain that occurs in piriformis syndrome being very similar at first to other types of pain that also cause sciatalgia, a number of differences exist. Similar to discogenic pains, the pain in piriformis syndrome spreads through the hip and the sciatic nerve path; unlike discogenic pains, weakness and numbness are rarely observed. Recreating the pain with maneuvers that place the sciatic nerve under stress during the physical examination can help lead the clinician to a diagnosis ${ }^{3,6,13}$. In piriformis syndrome, patients also experience pain during walking or prolonged sitting. Despite symptoms related to the straight-leg lifting test being diverse and contradictory in PS, they are usually normal, with the internal rotation of the hip exacerbating the symptoms ${ }^{4,13}$.

Imaging methods are used to rule out other conditions that might be confused with the syndrome ${ }^{6}$. MR imaging is particularly valuable in terms of showing nerve compression due to piriformis hypertrophy on the affected side 6 . While electromyography can be normal, it can be useful for diagnosis in long-term compression ${ }^{14}$. In the case of our patient, the presence of high fever; increased tenderness in the gluteal region with palpation; increased pain in the hip with flexion, abduction, and rotation; and elevated WBC count in addition to the typical clinical symptoms of sciatalgia led us to search for a preliminary diagnosis other than discopathy. At that point, the patient underwent tomography and then (due to the results of tomography) MR imaging.

Treatment of piriformis syndrome is related to its etiology. Medical treatment, physical therapy, acupuncture, therapeutic perisciatic block, botulinum toxin injection, or surgical intervention are among the potential treatment options. Abscesses due to pyomyositis are treated with percutaneous drainage or open surgery accompanied by ultrasound or computed tomography (CT) imaging ${ }^{10}$. In our case, surgical drainage and antibiotherapy was applied to the abscess inside the muscle because secondary piriformis syndrome had developed. In the culture performed, S. aureus grew, in accordance with the literature. 


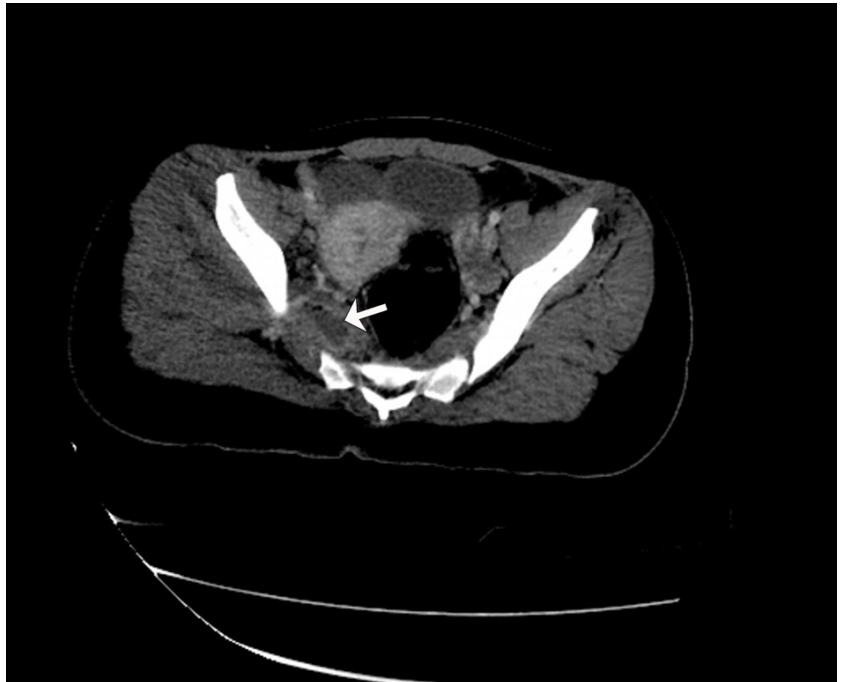

Figure 1: CT scan of pelvis demonstrate heterogeneity consistent with an abscess in the piriformis muscle on the right (arrow).

\section{Conclusion}

In patients who report to the emergency department with symptoms of sciatalgia, the focus of the differential diagnosis should be on maneuvers in the physical examination that place the piriformis muscle under stress. If piriformis syndrome due to pyomyositis is overlooked by emergency clinicians with sciatalgia in mind, it can lead to death or the need for high-cost treatment methods.

\section{Conflict of interest:}

The authors have no conflict of interest to declare

\section{Financial Disclosure:}

The authors declared that this study has received no financial support

The written consent form is taken from the patient.

"The case report has written in an anonymous characteristic, thus secret and detailed data about the patient has removed. Editor and reviewers can know and see these detailed data. These data are backed up by editor and by reviewers."

\section{References}

1. Serinken M, Eken C, Gungor F, Emet M, Al B. Comparison of Intravenous Morphine vs Paracetamol in Sciatica: A Randomized Placebo Controlled Trial. Acad Emerg Med. 2016.

2. Cansever T, Kabatas S, Ilgaz Ö, Yilmaz C, Caner H. Piriformis sendromuna genel bakis. Türk Nöroşirürji Dergisi. 2010;20 (1):30-5 .
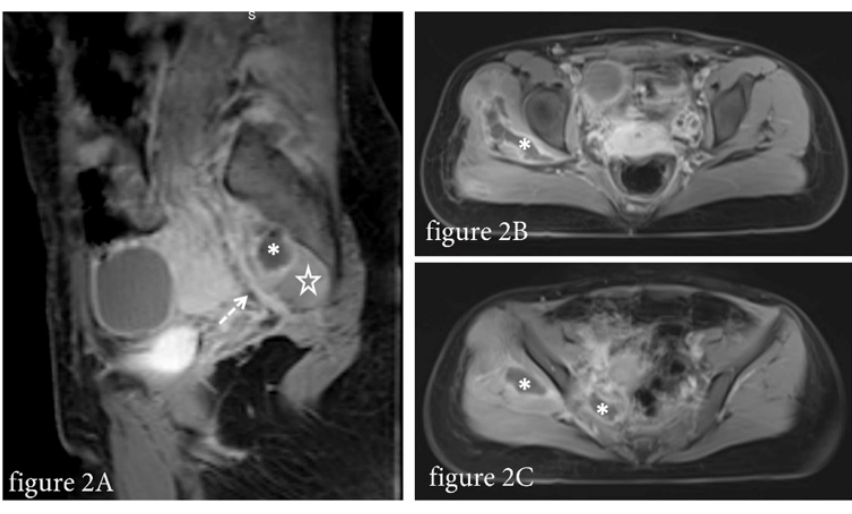

Figure 2: Sagittal (A), and axial (B, C) contrast enhanced (fat saturated) T1 weighted MR images show abscess formations in musculus piriformis and gluteal muscles (asterisks) and sagittal MR image (A) also shows compressed nervus ischiadicus (dashed arrow) below the abscess formation in the piriform muscle (star) (musculus piriformis syndrome).

3. Rossi P, Cardinali P, Serrao M, Parisi L, Bianco F, De Bac S. Magnetic resonance imaging findings in piriformis syndrome: a case report. Arch Phys Med Rehabil. 2001;82(4):519-21.

4. Byrd JT. Piriformis syndrome. Operative Techniques in Sports Medicine. 2005;13(1):71-9.

5. Boyajian-O'Neill LA, McClain RL, Coleman MK, Thomas PP. Diagnosis and management of piriformis syndrome: an osteopathic approach. J Am Osteopath Assoc. 2008;108(11):65764.

6. Michel F, Decavel P, Toussirot E, Tatu L, Aleton E, Monnier G, et al. The piriformis muscle syndrome: an exploration of anatomical context, pathophysiological hypotheses and diagnostic criteria. Ann Phys Rehabil Med. 2013;56(4):300-11.

7. Cassidy L, Walters A, Bubb K, Shoja MM, Tubbs RS, Loukas M. Piriformis syndrome: implications of anatomical variations, diagnostic techniques, and treatment options. Surg Radiol Anat. 2012;34(6):479-86.

8. Colmegna I, Justiniano M, Espinoza LR, Gimenez CR. Piriformis pyomyositis with sciatica: an unrecognized complication of "unsafe" abortions. J Clin Rheumatol. 2007;13(2):87-8.

9. Toda T, Koda M, Rokkaku T, Watanabe H, Nakajima A, Yamada $T$, et al. Sciatica caused by pyomyositis of the piriformis muscle in a pediatric patient. Orthopedics. 2013;36(2):e257-9.

10. Miller NJ, Duncan RD, Huntley JS. The conservative management of primary pyomyositis abscess in children: case series and review of the literature. Scott Med J. 2011;56(3):i-181.

11. Chiedozi LC. Pyomyositis. Review of 205 cases in 112 patients. Am J Surg. 1979;137(2):255-9.

12. Chong KW, Tay BK. Piriformis pyomyositis: a rare cause of sciatica. Singapore medical journal. 2004;45(5):229-31.

13. Kulcu DG, Naderi S. Differential diagnosis of intraspinal and extraspinal non-discogenic sciatica. J Clin Neurosci. 2008;15(11):1246-52.

14. Kraus E, Tenforde AS, Beaulieu CF, Ratliff J, Fredericson M. Piriformis syndrome with variant sciatic nerve anatomy: a case report. PM R. 2016;8(2):176-9. 